\title{
BMJ Open Quality Reducing prolonged chemoradiation treatment times for cervical cancer
}

\author{
Lucas Vitzthum, Jianling Yuan, Daniel Jones, Anne Boldt, Kathryn Dusenbery
}

To cite: Vitzthum L, Yuan J, Jones $\mathrm{D}$, et al. Reducing prolonged chemoradiation treatment times for cervical cancer. BMJ Open Quality 2019;8:e000516. doi:10.1136/ bmjoq-2018-000516

Portions of this work were presented in poster format at the American Society of Therapeutic Radiation Oncology Annual Meeting, 15 September 2014, San Francisco, California, USA.

Received 28 August 2018

Revised 9 August 2019

Accepted 18 August 2019

A) Check for updates

(C) Author(s) (or their employer(s)) 2019. Re-use permitted under CC BY-NC. No commercial re-use. See rights and permissions. Published by BMJ.

Department of Radiation Oncology, University of Minnesota, Minneapolis, Minnesota, USA

Correspondence to Dr Kathryn Dusenbery; dusen001@umn.edu

\begin{abstract}
Prolonged total treatment times (TTTs) beyond 56 days are associated with worse outcomes for cervical cancer treated with radiation therapy. We reviewed treatment times in a cohort of 24 consecutive patients treated with definitive chemoradiation (CRT) at our institution and found that only 14 patients $(58.3 \%)$ completed treatment in less than or equal to 56 days. The primary objectives of this institutional quality improvement initiative were to identify sources for delays in treatment completion and to implement effective measures in an effort to minimise prolonged TTT. Pareto plot and process mapping were used to identify and resolve root causes of prolonged treatment. The Plan-Do-Study-Act method was then implemented to reduce treatment duration. Postintervention treatment times were prospectively evaluated in 81 subsequent patients treated with definitive CRT. Process mapping identified inefficiencies with scheduling, staggered treatments and inadequate patient and staff education. Institutional changes were implemented, heavily utilising oncology nurses' skill set in staff re-education and care coordination. Our workflow was redesigned to reduce/eliminate treatment delays. These interventions led to a significant improvement in the percentage of patients meeting the goal TTT compared with the pre-intervention cohort $(85.2 \%$ vs $58.3 \%, p<0.01)$, and results were sustainable in additional 47 patients prospectively followed subsequently, potentially making a positive impact on their treatment outcomes.
\end{abstract}

\section{PROBLEM}

Cervical cancer is the third most common gynaecologic cancer in developed countries and the second most common cancer in females in developing countries. ${ }^{1}$ While early-stage cervical cancer may be cured with surgery alone, many women present with locoregionally advanced disease. The standard of care for higher-stage cervical cancer consists of external beam radiation therapy (EBRT) with concurrent chemotherapy followed by a brachytherapy (BT) boost to the cervix. ${ }^{2}$ It has been well established that prolonged treatment times from the start to finish of definitive chemoradiation (CRT) are associated with increased cancer recurrence and decreased survival. ${ }^{3-8}$ Despite the accepted importance of treatment duration, completing treatment in a timely fashion still faces practical challenges.
As a National Cancer Institute (NCI)-designated comprehensive cancer centre and the only university-based academic medical centre in the state, our institution has become the main referral site for women diagnosed with advanced cervical cancer, serving not only the metropolitan area, but also patients from rural Minnesota and bordering states. Although a departmental policy was in place to keep the total treatment time (TTT) to 56 days or less, as per Radiation Therapy Oncology Group protocols, an initial informal audit showed a disappointingly low compliance rate. ${ }^{9}$ Our aims were, thus, to identify reasons of treatment prolongation, to implement quality improvement (QI) measures and to compare TTT before and after these implementations among cervical cancer patients treated with definitive CRT.

\section{BACKGROUND}

In the USA, approximately 13000 new cases of cervical cancer are diagnosed annually with 4100 cancer-related deaths. ${ }^{10}$ CRT has been the standard of care for locoregionally advanced disease since 1999 when several randomised trials showed a survival advantage with the addition of concurrent cisplatin-based chemotherapy to radiation therapy (RT).$^{11} 12$ The treatment regimen consists of EBRT with concurrent weekly cisplatin followed by a BT boost. ${ }^{213}$ EBRT is typically delivered in 25-28 fractions, 5 days per week, over 5-5.5 weeks. BT is administered over 3-5 fractions, at least 48 hours apart, either interdigitated with or after the EBRT. Patients with parametria or sidewall disease may also require an additional EBRT boost, given on days without BT treatment.

TTT, defined as the time from first radiation fraction to treatment completion date, has been shown to significantly impact patient outcomes following definitive radiotherapy. In the era when radiation was used alone, treatment times beyond 7-9 weeks were shown to reduce locoregional control (LRC) and overall survival. ${ }^{3-8}$ Similar findings have been reported for patients treated with 
CRT. ${ }^{14-16}$ In one series, treatment times over 56 days were associated with a $17 \%$ increase in pelvic failures compared with shorter treatment times. ${ }^{14}$ Each extra day of treatment beyond 6-8 weeks can result in a loss of $0.6 \%$ per day in pelvic control. ${ }^{16}$ While the mechanism is not fully understood, it is hypothesised that an increased proliferation of tumour cells in response to treatment-induced cell killing through a phenomenon known as clonogenic accelerated repopulation is responsible for the decreased tumour control with prolonged treatment. ${ }^{17}$ In addition to worse clinical outcomes, prolonged treatment times have also been shown to significantly reduce overall patient satisfaction. ${ }^{18}$

Despite the recognised importance of TTT, completing treatment under 56 days can be challenging. We set out to review TTT for patients treated with definitive CRT at our institution, to identify sources of delays in treatment and to implement QI measures to enhance the delivery of care.

\section{BASELINE MEASUREMENT}

In the initial phase of the study, we sought to categorise patient and treatment characteristics and assess the prevalence of prolonged treatment time at our institution. After obtaining institutional review board approval, we retrospectively reviewed 24 consecutive patients treated with definitive CRT using a combination of EBRT and high-dose-rate (HDR) BT from July 2011 to August 2013. Only patients treated with curative intent were included.

Radiation treatment characteristics of these patients are presented in table 1. All patients were treated with EBRT followed by image-guided HDR tandem and ring (T\&R) $(\mathrm{n}=17,70.8 \%)$ or interstitial BT $(\mathrm{n}=7,29.2 \%)$ applications. Before starting BT, patients were usually required to undergo a diagnostic MRI to assess treatment response to EBRT and to allow selection of appropriate boost technique. A Smit sleeve was sewn into the endocervical canal in all patient receiving T\&R BT to facilitate applicator insertion. The median number of BT treatments was five with T\&R administered at least 48 hours between fractions, whereas interstitial BT was delivered in a two times per day fashion over 3 days. A total of 21 patients $(87.5 \%)$ received a parametrial boost $(\mathrm{PB})$ via anterior-posterior split-pelvic (SP) fields that occurred before, during or after BT. The majority of patients received EBRT at our university main campus $(\mathrm{n}=20,83.3 \%)$; however, patients were also treated at university affiliate sites $(n=2,8.3 \%)$ and outside institutions $(\mathrm{n}=2,8.3 \%)$, and subsequently came to the university for BT.

Concurrent chemotherapy with weekly cisplatin was given in all patients staged FIGO IB2 or higher. For patients treated with chemotherapy, $15(65.2 \%)$ received all five planned weekly doses. Chemotherapy was withheld in the remaining patients primarily due to cytopenia, and occasionally for severe nausea, dehydration and diarrhoea, at the discretion of the treating gynaecologic oncologist.

The median TTT among the 24 pre-intervention patients was 54 days (range 40-69 days). Of these, 14
Table 1 Radiation therapy treatment characteristics of the pre-intervention cohort

\begin{tabular}{ll}
\hline Treatment characteristics & \\
\hline EBRT & Median (range) \\
\hline Dose (Gy) & $45(45-45.5)$ \\
\hline \# of fractions & $25(25-26)$ \\
\hline EBRT field & $\mathrm{N}(\%)$ \\
\hline WP & $14(58.3 \%)$ \\
\hline EFRT & $10(41.7 \%)$ \\
\hline EBRT modality & $\mathrm{N}(\%)$ \\
\hline IMRT & $21(87.5 \%)$ \\
\hline 3D-CRT & $3(12.5 \%)$ \\
\hline Location of EBRT & $\mathrm{N}(\%)$ \\
\hline University campus & $20(83.3 \%)$ \\
\hline University affiliates & $2(8.3 \%)$ \\
\hline Outside institutions & $2(8.3 \%)$ \\
\hline Brachytherapy & Median (range) \\
\hline Dose (Gy) & $27.5(21-30)$ \\
\hline Fractions (T\&R only) & $5(5-6)$ \\
\hline Brachytherapy modality & $\mathrm{N}(\%)$ \\
\hline T\&R & $17(70.8 \%)$ \\
\hline Interstitial & $7(29.2 \%)$ \\
\hline Parametrial boost & Median (range) \\
\hline Dose (Gy) & $5.4(5-10.8)$ \\
\hline \# of fractions & $3(2-5)$ \\
\hline \# boosted & $21(87.5 \%)$ \\
\hline No boost & $3(12.5 \%)$ \\
\hline$R T$ teatnentch
\end{tabular}

RT treatment characteristics for the initial cohort of 24 patients treated with definitive CRT.

CRT, chemoradiation; 3D-CRT, three-dimensional conformal radiation therapy; EBRT, external beam radiation therapy; EFRT, extended field radiation therapy; IMRT, intensity-modulated radiation therapy; $\mathrm{RT}$, radiation therapy; T\&R, tandem and ring; WP, whole pelvis.

(58.3\%) had TTT $\leq 56$ days, whereas 10 (41.7\%) exceeded 56 days. With an approximately $40 \%$ baseline rate of failing to achieve target TTT, QI efforts were initiated with the goal of reducing this rate by at least half.

\section{DESIGN}

A QI team was established, comprising of physicians who specialise in gynaecologic malignancies, a resident physician as well as an oncology nurse.

A root cause analysis (RCA) was performed by the attending and resident physicians to determine the cause of treatment delay in the retrospective cohort. Manual chart review of the electronic health record (EHR) was performed to identify the primary cause of delay. For some patients, it was difficult to ascertain a primary cause for delay from the EHR alone. In these cases, members of the patient's treatment team were contacted for insight into 


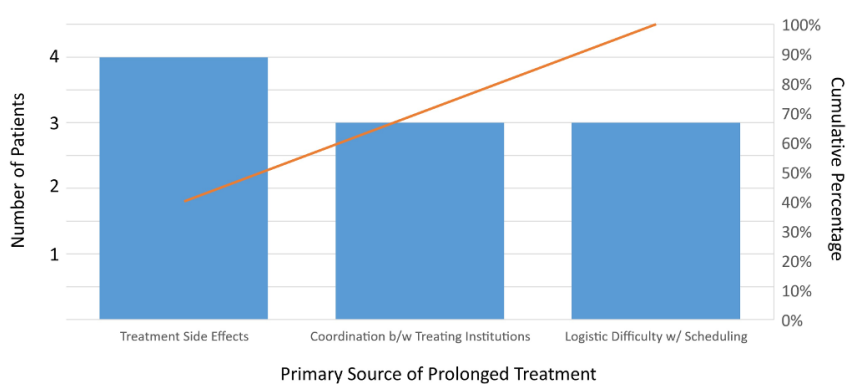

Figure 1 Pareto plot illustrating the primary causes of treatment prolongation among the 10 patients with TTT greater than 56 days. Patients treated prior to June 2013 represent the pre-intervention cohort. Quality improvement interventions were implemented from July 2013 to July 2017. Patients treated after July 2017 represent the postintervention cohort. b/w, between; TT, total treatment time; w/, with.

the cause of the prolonged treatment. Of the 10 patients who had TTT greater than 56 days, the primary causes of prolonged treatment could be assigned to three general categories, which is represented in a Pareto plot (figure 1). Four patients experienced treatment breaks due to toxicity, including cytopenia requiring transfusions and severe nausea, vomiting and dehydration necessitating inpatient fluid resuscitation. One of these patients was admitted to an outside community hospital, which resulted in further delays due to logistic challenges. Three patients had treatment prolongation due to gaps between the end of EBRT at an outside institution and the start of BT at our institution. Another three patients had prolonged treatment times because of difficulties scheduling one or more phases of their RT treatment. This group included one patient who had difficulty making treatment appointments due to a lack of reliable transportation.

We used a process map to visually display the workflow of how a patient is scheduled for treatment. Process mapping of our workflow revealed several potential sources for delays that were not apparent prior to creating a formal diagram of our workflow. The established protocol for the first fraction of BT included Smit sleeve placement and applicator insertion in the operating room (OR) followed by a planning MRI, a treatment planning CT, creation of the first BT plan and treatment delivery, all occurring in the same day. Such a long process necessitated the first OR timeslot of the morning, which was in high demand and required booking weeks in advance. Process mapping also revealed that the PB via split pelvis field was often added on after BT was complete, instead of intercalated into the BT schedule, thus prolonging TTT. For patients receiving EBRT at an outside facility who were referred to the university for BT, a stalling point was found in the timely scheduling of consultation appointment and BT procedures with a university radiation oncologist. Finally, this exercise showed that there was a knowledge gap among scheduling staff. Many of the medical assistants and schedulers were not aware that reducing treatment times was a priority in the treatment of cervical cancer, and therefore were not making a conscious effort to expedite the coordination of care.

To reduce treatment prolongation, we implemented improvement initiatives using Plan-Do-Study-Act (PDSA) cycles over the course of 4 years. To evaluate the efficacy of our new action plan, a post-intervention analysis of treatment times was prospectively conducted on 81 subsequent cervical cancer patients treated with definitive CRT and compared with that of the pre-intervention cohort using the $\chi^{2}$ test. The sustainability of our intervention was tested by evaluating an additional 47 patients after all PDSA cycles had been implemented and the formal QI project had ended.

\section{STRATEGY}

\section{PDSA cycle 1}

Plan: Our plan was to remind staff members within the department and at outside referring institutions about the impact of treatment time on patient outcome and their important roles in keeping the TTT under 56 days. We also planned to provide more education for patients about the importance of minimising unintentional treatment breaks during their initial consultation appointment.

Do: During the education process, subjective feedback revealed that staff and patients had not previously understood the impact of treatment time on cervical cancer outcomes.

Study: Even with education, we found that efforts in expediting care coordination and appointment scheduling were not consistent among the scheduling clerks. Some patients still missed treatment appointments leading up to BT.

Act: We created visual reminders to be posted near the scheduling desks and throughout the department and sent to referring facilities. Importance of minimising unplanned treatment breaks was not only presented to the patient during an initial consultation but reinforced during weekly on-treatment visits throughout the treatment course.

\section{PDSA cycle 2}

Plan: We planned to examine and redesign our workflow in an effort to reduce/eliminate treatment delays. We also sought to establish collaborative relationships with the outside providers to encourage referral of patients early in the course of EBRT so that elapsed time between EBRT and BT could be minimised.

Do: We reached out to OR scheduling team to leverage more first-case slots for our patients. We lobbied outside physicians to place referral orders early in course of patients' EBRT. Furthermore, we asked the physicians to declare an intention for a $\mathrm{PB}$ at the time of initiation of EBRT, rather than towards the end.

Study: We found that while OR was supportive of our efforts, reserving more first-case start time was not always achievable, and thus separating the Smit sleeve placement from first insertion of T\&R may be necessary. In some 


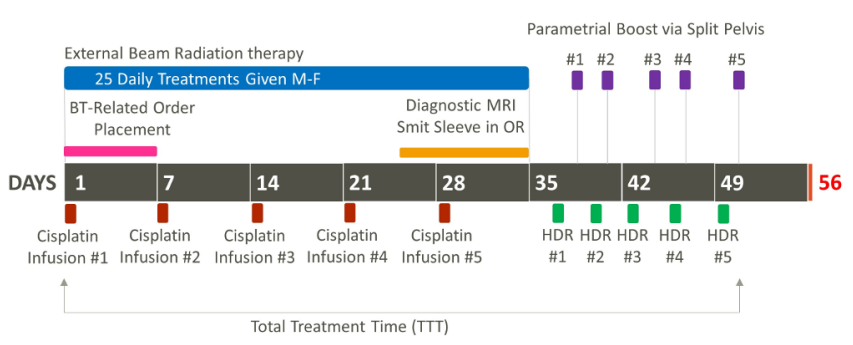

Figure 2 Re-designed workflow and timeline of a cervical cancer treatment course incorporating external beam radiation therapy with concurrent weekly cisplatin, five fractions of brachytherapy (BT) and up to five fractions of a parametrial boost that finishes with a TTT of less than 56 days. HDR, high-dose rate; OR, operating room.

instances, OR or BT slots could not be secured due to lack of timely orders from physicians. For patients requiring $\mathrm{PB}$, it could be a source of delay when it is given after all five BT were complete.

Act: In order to make more OR times workable, Smit sleeve placement was scheduled on a separate day prior to first BT. On the actual days of BT, T\&R applicators could be inserted in the clinic or sedation suite, significantly reducing the demand for $\mathrm{OR}$. Additionally, for patients requiring $\mathrm{PB}$, the delivery of $\mathrm{SP}$ fields was shifted from following BT to interdigitating with BT on days BT was not given. All physicians at our institution were also asked to place BT-related orders within the first 5 days of initiating EBRT to allow more flexibility in scheduling with OR for Smit sleeve placement, sedation suite for applicator insertion and radiology for treatment planning MRI. The improved workflow is shown in figure 2.

\section{PDSA cycle 3}

Plan: While our initial QI efforts were successful, we recognise that sustainability is important to have a longterm impact on patient outcome. In this final PDSA cycle, we planned to ensure ongoing accountability.

Do: We created a nurse-led database to prospectively track all cervical cancer patients treated with curative intent. Nurses in the department routinely reviewed the progress of on-treatment patients at least once a week and functioned as a liaison between patients, physicians and schedulers.

Study: Of the additional 47 patients in the sustainable phase of the study, the number of patients exceeding the goal TTT of 56 days were kept at a minimum (see section Results).

Act: We will maintain our current QI efforts. Furthermore, any prolonged TTT will trigger an RCA and ensure continuous improvement.

\section{RESULTS}

QI interventions began in August of 2013. Of the subsequent 81 consecutive cervical cancer patients treated with definitive CRT from 2013 to 2017, the median TTT was 52 days (range 37-77 days). Compared with only $58.3 \%$ pre-intervention patients finishing treatment in $\leq 56$ days,
$85.2 \%$ (69 out of 81 ) of the post-intervention patients were able to achieve TTT goals, a statistically significant $(\mathrm{p}<0.01)$ improvement (figure 3$)$. Among the 12 patients with TTT over 56 days in the post-intervention cohort, the primary cause of the delay included treatment-related side effects $(n=4)$, delayed transfer from an outside institution $(n=3)$, repeat patient no shows $(n=3)$, split pelvis EBRT delivered after BT $(n=1)$ and delayed OR clearance $(n=1)$.

This QI has led to sustainable results with 43 of 47 patients $(91 \%)$ finishing within 56 days or less during the next 18 months since the completion of additional interventions in 2017. Of the four patients with delayed treatment times, one had to have an unplanned surgery following EBRT and one developed delirium during interstitial BT, necessitating discontinuation and rescheduling of the remaining treatment. Another patient experienced prolongation of 1 day due to unavailability of sterilised BT equipment. The final patient with complex social issues missed multiple treatments despite repeated efforts in education and rescheduling. There are essentially no instances of preventable delays.

\section{Lessons and limitations}

Our RCA showed that the primary reasons for prolonged TTT included treatment-related toxicities necessitating treatment break, in-house scheduling delays and transfer delays from outside referring institutions. Petereit et al reported similar findings with the most commonly observed causes for treatment delay being RT-related side effects and scheduling gaps between EBRT and BT. ${ }^{8}$ These analyses suggest that while some patients may have unpredictable delays due to side effects, a significant portion of treatment prolongation can be avoided by making systematic changes to institutional practices, such as scheduling.

Process mapping each step of the RT treatment process revealed several opportunities for intervention. Institutional changes were made regarding the timing of Smit sleeve placement in relation to the first BT, more efficient delivery of PB and early scheduling efforts by physicians and staff. This exercise also revealed a need to educate all members of the treatment team, including in-house schedulers as well as those at the outside referring facilities.

Our QI interventions led to a significant increase in the proportion of patients $(85 \%)$ able to complete treatment within 56 days, potentially making a positive impact on their treatment outcomes. Treatment-related side effects and delayed transfers from outside institutions remained common causes for prolonged TTT. We worked to further reduce delays from treatment side effects by encouraging patients to report symptoms early and more proactively managing dehydration, nausea and diarrhoea. Our results were sustained with $91 \%$ patients achieving the target TTT of 56 days among the additional 47 prospectively followed patients after all inventions had been implemented. We found that the process of 


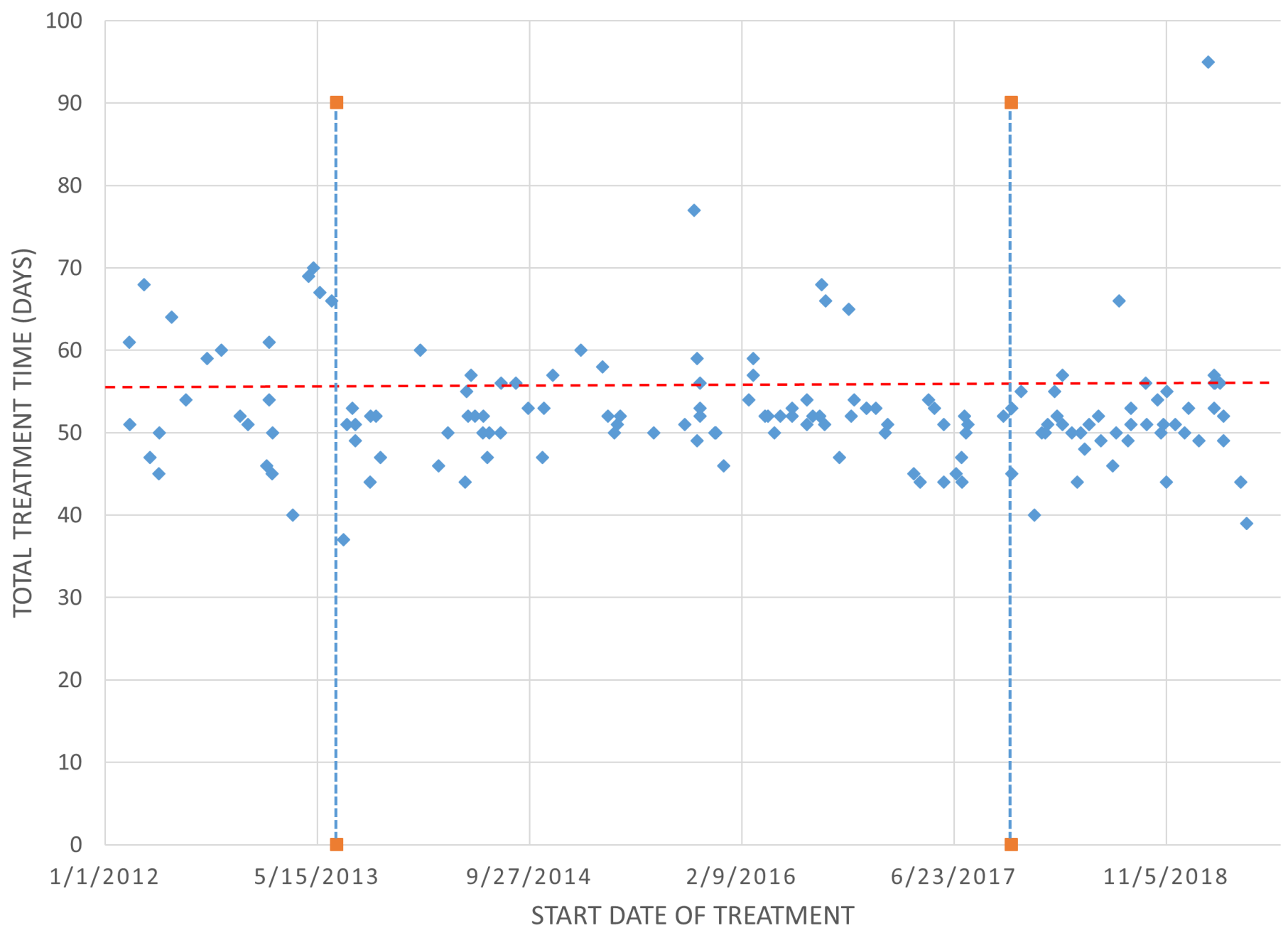

Figure 3 Run chart showing total treatment times (TTTs) pre-implementing and post-implementing quality improvement (QI) interventions. Dotted horizontal red line represents goal TTT of $\leq 56$ days. Dashed blue dotted line represents the final implementation date for our QI interventions.

formally analysing the clinically relevant endpoint of TTT was helpful in understanding our clinical workflow and believe that it will improve patient care.

This project was conducted at an academic medical centre with NCI comprehensive cancer centre designation. Chemotherapy was administered through the department of gynaecologic oncology, while RT was administered by the department of radiation oncology. We believe that the lessons from this study will be applicable to similar tertiary care centres. Centres with less resources will likely face an even greater challenge in coordinating care and providing supportive measures to prevent prolonged TTT.

\section{CONCLUSION}

Prolonged treatment times have been associated with decreased LRC in cervical cancer patients treated with definitive CRT. ${ }^{14-16}$ TTT for cervical cancer patients treated with definitive CRT at our institution was found to be suboptimal with only $58 \%$ of patients completing in $\leq 56$ days. Preventable delays were attributed to inefficient OR scheduling, lack of education and unnecessarily staggered RT treatment. Points of intervention were identified using process mapping. Effective measures were implemented which improved workflow and increased the number of patients with an acceptable TTT to $\sim 90 \%$. Treatment-related side effects and delayed transfers from outside institutions remained common causes for prolonged TTT and will be investigated for further improvement approaches.

Acknowledgements The authors would like to thank the physicians, nurses and support staff at our institution for their help in implementing this project.

Contributors LV contributed to the project design, implementation, data collection, data analysis and manuscript preparation. JY contributed to the project design, Ql implementations, manuscript preparation and editing. AB contributed to data collection, QI implementations, Ql brainstorming and manuscript editing. DJ contributed to the project design, implementation and manuscript editing. $\mathrm{KD}$ contributed to the project conception and design, staff interviewing, QI implementations, manuscript writing and editing.

Funding The authors have not declared a specific grant for this research from any funding agency in the public, commercial or not-for-profit sectors.

Competing interests None declared.

Patient consent for publication Not required.

Provenance and peer review Not commissioned; externally peer reviewed. 
Open access This is an open access article distributed in accordance with the Creative Commons Attribution Non Commercial (CC BY-NC 4.0) license, which permits others to distribute, remix, adapt, build upon this work non-commercially, and license their derivative works on different terms, provided the original work is properly cited, appropriate credit is given, any changes made indicated, and the use is non-commercial. See: http://creativecommons.org/licenses/by-nc/4.0/.

\section{REFERENCES}

1. Torre LA, Bray F, Siegel RL, et al. Global cancer statistics, 2012. CA Cancer J Clin 2015;65:87-108.

2. Dorigo O, Eifel PJ, Fisher CM, et al. NCCN cervical cancer guidelines version 1.2018. published online first:, 2018. Available: https://www. nccn.org/professionals/physician_gls/pdf/cervical.pdf [Accessed 24 May 2018].

3. Chatani M, Matayoshi Y, Masaki N, et al. High-Dose rate intracavitary irradiation for carcinoma of the uterine cervix. Strahlenther Onkol 1997;173:379-84.

4. Fyles A, Keane TJ, Barton M, et al. The effect of treatment duration in the local control of cervix cancer. Radiother Oncol 1992;25:273-9.

5. Girinsky T, Rey A, Roche B, et al. Overall treatment time in advanced cervical carcinomas: a critical parameter in treatment outcome. Available: redjournal.orghttp://www.redjournal.org/article/0360-3016( 93)90522-W/pdf [Accessed 21 May 2018].

6. Lanciano RM, Pajak TF, Martz K, et al. The influence of treatment time on outcome for squamous cell cancer of the uterine cervix treated with radiation: a patterns-of-care study. Int $J$ Radiat Oncol Biol Phys 1993;25:391-7.

7. Perez CA, Grigsby PW, Castro-Vita H, et al. Carcinoma of the uterine cervix. I. impact of prolongation of overall treatment time and timing of brachytherapy on outcome of radiation therapy. Int $J$ Radiat Oncol Biol Phys 1995;32:1275-88.

8. Petereit DG, Sarkaria JN, Chappell R, et al. The adverse effect of treatment prolongation in cervical carcinoma. Int J Radiat Oncol Biol Phys 1995;32:1301-7.
9. Schefter T, Winter K, Kwon JS, et al. RTOG 0417: efficacy of bevacizumab in combination with definitive radiation therapy and cisplatin chemotherapy in untreated patients with locally advanced cervical carcinoma. Int J Radiat Oncol Biol Phys 2014;88:101-5.

10. Siegel R, Ward E, Brawley O, et al. Cancer statistics, 2011: the impact of eliminating socioeconomic and racial disparities on premature cancer deaths. CA Cancer J Clin 2011;61:212-36.

11. Morris M, Eifel PJ, Lu J, et al. Pelvic radiation with concurrent chemotherapy compared with pelvic and para-aortic radiation for high-risk cervical cancer. $N$ Engl J Med Overseas Ed 1999;340:1137-43.

12. Rose PG, Bundy BN, Watkins EB, et al. Concurrent cisplatin-based radiotherapy and chemotherapy for locally advanced cervical cancer. N Engl J Med 1999;340:1144-53.

13. Viswanathan AN, Beriwal S, De Los Santos JF, et al. American brachytherapy Society consensus guidelines for locally advanced carcinoma of the cervix. Part II: high-dose-rate brachytherapy. Brachytherapy 2012;11:47-52.

14. Song S, Rudra S, Hasselle MD, et al. The effect of treatment time in locally advanced cervical cancer in the era of concurrent chemoradiotherapy. Cancer 2013;119:325-31.

15. Tanderup K, Fokdal LU, Sturdza A, et al. Effect of tumor dose, volume and overall treatment time on local control after radiochemotherapy including MRI guided brachytherapy of locally advanced cervical cancer. Radiother Oncol 2016;120:441-6.

16. Mazeron R, Castelnau-Marchand P, Dumas I, et al. Impact of treatment time and dose escalation on local control in locally advanced cervical cancer treated by chemoradiation and imageguided pulsed-dose rate adaptive brachytherapy. Radiother Oncol 2015;114:257-63.

17. Huang Z, Mayr NA, Gao M, et al. Onset time of tumor repopulation for cervical cancer: first evidence from clinical data. Int $J$ Radiat Oncol Biol Phys 2012;84:478-84.

18. Famiglietti RM, Neal EC, Edwards TJ, et al. Determinants of patient satisfaction during receipt of radiation therapy. Int J Radiat Oncol Biol Phys 2013;87:148-52. 